\title{
Screening for adverse childhood experiences within paediatric patients in Rzeszów, Poland
}

\author{
Cassandra L. Mejia', Grzegorz Telega', Samantha L. Wilson', Malgorzata Nagorska² \\ ${ }^{1}$ Medical College of Wisconsin, Milwaukee, Wisconsin, United States \\ 2Institute of Experimental and Clinical Medicine, Medical Faculty, University of Rzeszow, Poland
}

\section{ABSTRACT}

Introduction: Adverse childhood experiences (ACEs) are stressful or potentially traumatic events correlated with negative, lasting effects on health.

Aim of the study: This study sought to assess: 1) prevalence of ACEs within a paediatric population in Rzeszów, Poland, 2) paediatrician screening practices for ACEs, and 3) physician experiences and opinions regarding ACE screening.

Material and methods: Caregivers $(N=110)$ of children aged 6 months to 18 years completed a translated Adverse Childhood Experiences Questionnaire (CYW ACE-Q), developed by the Centre for Youth Wellness, San Francisco. CYW ACE-Q scores were compared to physician perception of patient stress and need for intervention. Physicians $(N=18)$ provided additional opinions regarding ACE screening by completing a Physician Experiences in ACEs Questionnaire (PEA-Q).

Results: CYW ACE-Q responses revealed $27.3 \%$ of patients scored $\geq 4$ ACEs, a positive screening reflecting increased concerns for toxic stress. An additional $30 \%$ of patients experienced 1-3 ACEs. In $14.5 \%$ of patients, screening was positive but physician perception of stress was negative. Of the patients with a positive screening, $53.3 \%$ had discordant positive physician perception of stress and only $46.6 \%$ were evaluated as in need of intervention. Physicians overestimated stress in patients with low parental education $(p=0.024)$ and from rural areas $(p=0.01)$ but underestimated stress in urban patients $(p=0.01)$. All surveyed physicians $(N=18)$ believed that ACEs were important in primary health; only $5 \%$ reported there was enough time to screen or resources to intervene and only 39\% felt confident discussing ACEs with patients and families.

Conclusions: A significant prevalence of ACEs was noted within this paediatric sample in Rzeszów, Poland. Results highlight the incremental benefit of ACE screening to supplement physician perception of family stress. Further research is needed to guide implementation and education of physicians on the practical aspects of ACE screening and intervention strategies.

\section{KEY WORDS:}

adolescent, child, paediatrics, child abuse, life change events.

\section{INTRODUCTION}

Adverse childhood experiences (ACEs) are stressful or potentially traumatic events occurring during a devel- opmental period (i.e. childhood or adolescence). Toxic stress response occurs when a child is continually exposed to prolonged adversity without protective factors or buffers (e.g. supportive relationship with caregiver)

\section{ADDRESS FOR CORRESPONDENCE:}

Cassandra L. Mejia, Medical College of Wisconsin, 8701 W Watertown Plank Rd, Milwaukee, WI 53226,

United States, e-mail: mejia.cass22@gmail.com 
leading to prolonged allostasis and a dysregulation of the physiological stress response. This leads to altered levels of hormones and neurotransmitters, which ultimately alters organ systems and brain architecture, increasing the risk of chronic disease later in life [1]. The Kaiser Permanente study examined >17,000 participants in California, USA from 1995 to 1997 and found that as the number of ACEs increased, the risk for developing stress-related disease later in life increased in a graded fashion [2]. This includes but is not limited to ischaemic heart disease, chronic lung disease, liver disease, obesity, cancer, depression, substance abuse, and suicide attempts [2].

Further research has linked ACEs with adverse health outcomes in Europe as well. A meta-analysis across eight Eastern European countries surveying young adults in secondary or higher education demonstrated a significant association between ACEs and health harming behaviours [3]. Furthermore, a cross-sectional study of students from five Polish universities $(N=1722)$ revealed that $19 \%$ of participants experienced four or more ACEs, which was associated with increased health-harming behaviours such as suicide attempts, self-harm, smoking, drug and alcohol abuse, and early sexual activity [4]. Collectively, research has shown the correlation of ACEs with health outcomes within Eastern Europe and Poland; however, current studies of ACEs in Poland consist of retrospective ACE screening of adults. One prospective longitudinal study of 6-8-year-old children in Poland $(N=291)$ revealed an association between recent stressful lifeful events, symptoms related to stress, and negative impacts on a health-related quality of life survey [5]. While this further supports the importance of stressful events on paediatric health, this study focused on recent stressful events and shorter health quality outcomes. There remains little research on the prevalence of ACEs within paediatric patients in Poland and clinical screening practices.

The purpose of this study was to explore the concept of ACEs within a paediatric population in Rzeszów Poland. Specific objectives included:

1) identifying the prevalence of ACEs in the paediatric population seen at Sokrates Clinic, Rzeszów, Poland,

2) paediatrician screening practices for ACEs and accuracy of stress identification,

3) exploring physician experiences and opinions regarding ACE screening within the following five categories:

- provider training and awareness of ACEs,

- perception and attitude of screening,

- perceived control over screening and intervention,

- perceived cultural acceptance of screening,

- perceived social approval of screening.

\section{MATERIAL AND METHODS}

Institutional Review Board (IRB) approval was obtained by both the University of Rzeszów and the Medical
College of Wisconsin. Over the course of three months (June-August 2016), 110 caregivers of patients aged 6 months to 18 years completed a demographic questionnaire and a translated version of the Adverse Childhood Experiences Questionnaire (CYW ACE-Q) developed by the Centre for Youth Wellness (CYW), San Francisco, CA [6], prior to their scheduled appointment at Sokrates Clinic in Rzeszów, Poland. This location was chosen because it served a diverse group of rural and urban patients. The CYW ACE-Q was handed to caregivers by a nurse or other medical staff, using a modified CYW sample introduction [7] and a recruitment letter outlining the study participation. Caregivers were assured that participation was completely voluntary and choosing to complete or not complete the questionnaire would not affect their medical care in any way. Returning the completed CYW ACE-Q in a sealed envelope to the medical staff acted as caregiver agreement to participate in the study. The CYW ACE-Q instructed the patient's caregiver to give the total number of ACEs that applied to the patient without identifying the specific ACEs. This acted as additional privacy protection to the anonymous questionnaire because a specific ACE could not be linked to the patients.

The CYW ACE-Qs were scored and interpreted based on CYW recommendations [7]. Without having access to the CYW ACE-Q, the patients' physicians completed a questionnaire assessing their perception of patient/family stress and need for intervention. CYW ACE-Q scores were compared to physician perception using chi square tests on SPSS software. Additionally, 18 physicians completed the Physician Experience with ACEs questionnaire (PEA-Q), eliciting their opinions and experiences in ACE screening.

The CYW ACE-Q [6] asked respondents to indicate the number (not the specific type) of the following adverse childhood experiences: 1) emotional abuse, 2) physical abuse, 3) sexual abuse, 4) emotional neglect, 5) physical neglect, 6) divorced or separated parents, 7) caregivers with substance abuse, 8) incarcerated caregiver/relative, 9) caregiver with a mental illness, 10) witnessing routine abuse of mother/caregiver 11) foster care, 12) bullying, 13) parent or guardian death, 14) deportation/immigration, 15) life-threatening illness, 16) neighbourhood violence, and 17) discrimination due to race, sexual orientation, place of birth, disability, or religion. A score of four or more ACEs was considered a positive screening, reflecting increased concerns for toxic stress. According to CYW recommendations, a score of 1-3 ACEs with clinical signs of stress was considered a positive screening as well. However, because this study did not objectively account for clinical signs, a score of 1-3 was considered subclinical, indicating that additional screening or monitoring may be appropriate.

Three questionnaires were created for the purposes of this study: Demographic, Physician Perception of Stress, and Physician Experiences with ACEs (PEA-Q). These questionnaires are available upon request from the first 
author. All study materials were translated into Polish and subsequently back-translated. Demographic questions included parental education (below high school, high school, college, graduate school), socioeconomic status (below average, average, above average), rural or urban living status, and family structure (single parent, married parents, divorced parents with shared custody, divorced parent with sole custody, single-sex partners) (Table 1). Additionally, caregivers were asked if they felt comfortable discussing ACEs with their physician.

Physician perception of stress and need for intervention was assessed by the following two questions:

1. Outside of the chief complaint, how much stress do you perceive this patient and family to be experiencing? (none/small amount/large amount)

2. Do you believe this patient is in need of additional resources or evaluation outside of immediate medical care? (yes/no)

CYW ACE-Q and physician perception were considered congruent as follows: 0 ACEs with "no stress", 1-3 ACEs with "small or large amount of stress", and $\geq 4$ ACEs with "large amount of stress". Comparing 1-3 ACEs with "small or large amount of stress" allowed physicians' perception of stress to account for both a subclinical screening of 1-3 ACEs ("small amount of stress") and a positive screening of 1-3 ACEs with observed clinical symptoms ("large amount of stress").

The Physician Experience with ACEs questionnaire (PEA-Q) was created in consultation with a US practicing physician, who is native to and completed medical school in Poland, increasing cultural relevance and improved translation of the addressed concepts. The questionnaire elicited physicians' opinions and experiences within the following five categories: 1) provider training and awareness of ACEs, 2) perception and attitude of screening, 3) perceived control over screening and intervention, 4) perceived cultural acceptance of screening, and 5) perceived social approval of screening.

\section{RESULTS}

CYW ACE-Q responses revealed that $27.3 \%$ of patients experienced four or more ACEs, a positive screening reflecting increased concerns for toxic stress (Fig. 1). An additional 30\% reported 1-3 ACEs (i.e. subclinical screening), indicating that additional monitoring may be appropriate. There were no significant differences between ACE scores with regard to parental education and economic status $(p=0.22)$. ACE scores were lower in patients from rural areas compared to urban areas in total number of ACEs $(p \leq 0.022)$. There was a significant relationship between family structure and the total amount of ACEs $(p \leq 0.003)$.

Compared to the CYW ACE-Q, physicians overestimated stress in patients with low parental education $(p=0.024)$ and from rural areas $(p=0.01)$ but under-
TABLE 1. Demographic characteristics of the studied paediatric population $(N=110)$

\begin{tabular}{|c|c|c|}
\hline Characteristic & Number $(n)$ & Percentage (\%) \\
\hline \multicolumn{3}{|l|}{ Age (years) } \\
\hline $1-4$ & 10 & 9 \\
\hline $5-9$ & 33 & 30 \\
\hline $10-13$ & 44 & 40 \\
\hline $14-17$ & 22 & 20 \\
\hline \multicolumn{3}{|l|}{ Location } \\
\hline Rural & 29 & 26 \\
\hline Urban & 75 & 68 \\
\hline \multicolumn{3}{|l|}{ Parental education } \\
\hline Below high school & 5 & 5 \\
\hline High school & 46 & 42 \\
\hline College & 52 & 47 \\
\hline Graduate school & 4 & 4 \\
\hline \multicolumn{3}{|l|}{ Economic status } \\
\hline Below average & 12 & 11 \\
\hline Average & 72 & 65 \\
\hline Above average & 24 & 22 \\
\hline \multicolumn{3}{|l|}{ Family structure } \\
\hline Single parent & 11 & 10 \\
\hline Married parents & 69 & 63 \\
\hline Divorced with shared custody & 17 & 15 \\
\hline Divorced with sole custody & 6 & 5 \\
\hline
\end{tabular}

TABLE 2. Total number of adverse childhood experiences vs. provider assessment of stress

\begin{tabular}{|l|l|c|c|}
\hline & & $\begin{array}{c}\text { Number } \\
(n)\end{array}$ & $\begin{array}{c}\text { Percentage } \\
(\%)\end{array}$ \\
\hline \multirow{2}{*}{ ACE 0 } & Provider in agreement & 47 & 42.7 \\
\cline { 2 - 4 } & Provider in disagreement & 0 & 0 \\
\hline \multirow{2}{*}{ ACE 1-3 } & Provider in agreement & 13 & 11.8 \\
\cline { 2 - 4 } & Provider in disagreement & 20 & 18.2 \\
\hline \multirow{2}{*}{ ACE $\geq 4$} & Provider in agreement & 14 & 12.7 \\
\cline { 2 - 4 } & Provider in disagreement & 16 & 14.5 \\
\hline
\end{tabular}

$A C E=0$ was compared with "no stress", $A C E=1-3$ was compared with "small or large amount of stress", ACE $\geq 4$ was compared with "large amount of stress"

estimated stress in urban patients $(p=0.01)$. Positive ACE-Q screening ( $\geq 4$ ACEs) with discordant physician perception of stress ("no stress or small amount of stress") occurred in $11 \%$ of patients; $18.2 \%$ of patients had $1-3$ ACEs with discordant physician perception of stress ("no stress"). Of the patients with a positive screening, 53.3\% had discordant positive physician perception of stress (Table 2 ) and only $46.6 \%$ were evaluated as in need of intervention (Table 3). Regarding caregiver comfort in discussing ACEs with their paediatrician, 25\% reported 
TABLE 3. Total number of adverse childhood experiences vs. provider intervention recommendation

\begin{tabular}{|c|c|c|c|}
\hline & & $\begin{array}{c}\text { Number } \\
(n)\end{array}$ & $\begin{array}{c}\text { Percentage } \\
(\%)\end{array}$ \\
\hline \multirow[t]{2}{*}{$\mathrm{ACE}<4$} & $\begin{array}{l}\text { No intervention } \\
\text { recommended }\end{array}$ & 63 & 57.3 \\
\hline & $\begin{array}{l}\text { Intervention } \\
\text { recommended }\end{array}$ & 17 & 15.5 \\
\hline \multirow[t]{2}{*}{$\mathrm{ACE} \geq 4$} & $\begin{array}{l}\text { No intervention } \\
\text { recommended }\end{array}$ & 16 & 14.5 \\
\hline & $\begin{array}{l}\text { Intervention } \\
\text { recommended }\end{array}$ & 14 & 12.7 \\
\hline
\end{tabular}

they would be comfortable, $25 \%$ would not, and $46 \%$ were unsure.

The surveyed physicians had been in practice for between two and 30 years, with an average of 9.3 years. Physician responses $(N=18)$ to PEA-Q revealed the average number of the 10 ACEs routinely asked about by physicians personally was six, with a range of $0-10$. The average number of ACEs routinely reported to the physicians by other members of the medical team was eight with a range of $1-10$. While $84 \%$ of physicians reported receiving adequate training in ACE screening, only 39\% of physicians felt confident discussing ACEs with patients and families. One hundred per cent of the surveyed physicians stated that ACEs were important in primary health care, and $88 \%$ reported that stressors in patients' homes affect their health. However, $71 \%$ of physicians reported there was not enough time to screen for ACEs in a primary care setting. Furthermore, only $5 \%$ of physicians reported that there were resources in the hospital to manage family stressors; an additional 44\% were unsure/neutral.

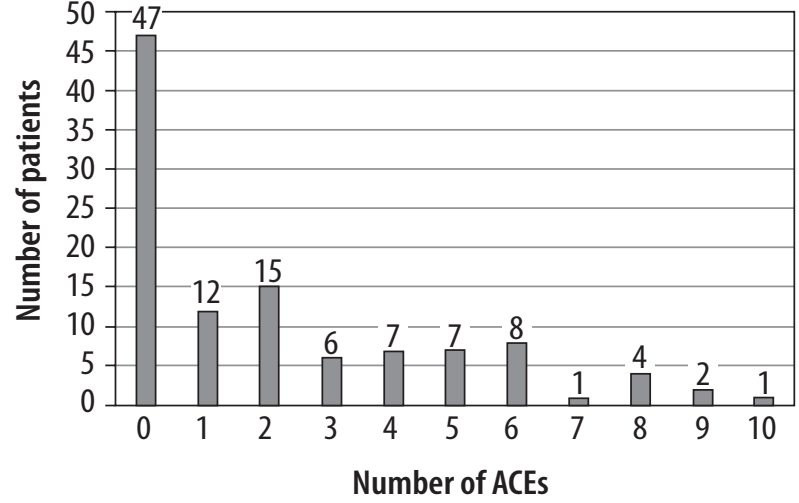

FIGURE 1. Total number of adverse childhood experiences (ACEs). Caregivers $(N=110)$ of children aged 6 months to 18 years completed a modified version of the CWY ACE- $Q$ instructing them to indicate the number of ACEs that applied to their child without identifying the specific ACEs

Sixty-seven per cent of surveyed physicians stated it was appropriate to discuss ACEs with patients and families. Furthermore, $61 \%$ expressed that it was a physician's role to ask about private family stressors, while $16 \%$ disagreed. Thirty-nine per cent of surveyed physicians reported that the leadership would approve of routine screening, with the remaining being neutral/unsure (Table 4).

\section{DISCUSSION}

A significant number of patients, $27.3 \%$, had a positive CYW ACE-Q screening ( $\geq 4$ ACEs). These patients are at risk for toxic stress and subsequent medical sequelae without proper assessment and intervention if indicated (e.g. lack of buffering relationship or other resilience factors).

TABLE 4. Physician $(N=18)$ responses to Physician Experiences in ACEs Questionnaire (PEA-Q)

\begin{tabular}{|l|c|c|c|c|c|}
\hline Statement & $\begin{array}{c}\text { Strongly } \\
\text { agree (\%) }\end{array}$ & $\begin{array}{c}\text { Agree } \\
(\%)\end{array}$ & $\begin{array}{c}\text { Neutral/ } \\
\text { unsure (\%) }\end{array}$ & $\begin{array}{c}\text { Disagree } \\
\text { (\%) }\end{array}$ & $\begin{array}{c}\text { Strongly } \\
\text { disagree (\%) }\end{array}$ \\
\hline $\begin{array}{l}\text { Medical experience prepared me to understand the effect of } \\
\text { adverse childhood experiences on patient health }\end{array}$ & 17 & 67 & 11 & 5 & 0 \\
\hline I do not feel confident asking about families' stressful experiences & 0 & 22 & 39 & 22 & 17 \\
\hline $\begin{array}{l}\text { It is important in primary health care to pay attention to adverse } \\
\text { childhood experiences }\end{array}$ & 11 & 89 & 0 & 0 & 0 \\
\hline Stressors at home do not affect the health of my patients & 5 & 5 & 5 & 33 & 50 \\
\hline $\begin{array}{l}\text { There are resources in my hospital to help families manage life } \\
\text { stressors }\end{array}$ & 0 & 5 & 44 & 33 & 17 \\
\hline $\begin{array}{l}\text { I do not ask about adverse childhood experiences because I am } \\
\text { unable to prevent them }\end{array}$ & 0 & 0 & 39 & 44 & 17 \\
\hline $\begin{array}{l}\text { There is not enough time to screen for adverse childhood expe- } \\
\text { riences in primary paediatric health care }\end{array}$ & 28 & 44 & 22 & 5 & 0 \\
\hline $\begin{array}{l}\text { It is appropriate to discuss adverse childhood experiences with } \\
\text { patients and their families }\end{array}$ & 11 & 56 & 33 & 0 & 0 \\
\hline It is not a physician's role to ask about private family stressors & 5 & 11 & 22 & 44 & 17 \\
\hline $\begin{array}{l}\text { The leadership of this medical centre would approve of routine } \\
\text { screening for adverse childhood experiences }\end{array}$ & 11 & 28 & 61 & 0 & 0 \\
\hline
\end{tabular}


Of the patients with positive ACE-Q screening, 53.3\% had a negative physician perception of stress and only $46.7 \%$ were considered to be in need of intervention. These data highlight the incremental benefit of ACE screening to supplement physician perception of family stress. Further indications for the need of supplemental ACE screening include overestimated stress in rural patients and those with low parental education as well as underestimated stress in urban patients. Currently, the reasons for this discrepancy are unclear. Because this health care clinic was located in the city of Rzeszów, a cultural disconnect between physicians and their rural patients and/or perceptual bias may contribute to this incongruity. Interestingly, ACE scores were overall lower in rural patients. Additional studies may help illuminate reasons for this discrepancy.

With almost half of caregivers unsure if they would be comfortable discussing ACEs with their paediatricians, increasing awareness of ACEs and adverse health outcomes along with normalising these conversations may promote more accurate screening. Further exploration of caregiver opinions of ACE screening is recommended for creating or adjusting screening tools and procedures that are accepted by caregivers.

The surveyed 18 physicians' responses to the PEA-Q provided insight into the barriers of routine ACE screening for the study population.

\section{PROVIDER TRAINING AND AWARENESS OF ACES}

The responses indicated that the majority of physicians believed their medical experience provided an adequate understanding of ACEs; however, less than half indicated that they were comfortable discussing ACEs within patient visits. This indicates a need for practical clinical training on initiating conversations about ACEs with patients and their families. Similarly, a study done in 2006 in Gizycko, Poland, revealed that only 25.1\% of surveyed patients $(n=1000)$ responded "always" to physicians advising on healthy living during primary care appointments, and only $2.1 \%$ and $1.2 \%$ responded "always" to physicians discussing family conditions and work conditions, respectively [8]. Furthermore, a study of Polish paediatric nurses $(N=160)$ showed that while $96.25 \%$ believed skills in identifying clinical signs of child maltreatment were important, only $12 \%$ felt confident in this identification [9]. Collectively, these studies support the need for education and improved practical skills in both screening for childhood traumatic events and discussing socioeconomic factors affecting health. Similarly, improved education on ACE screening has been recommended from studies of providers from the United States. For example, a study surveying family medicine residents $(N=112)$ showed that while $80 \%$ believed it was their role to screen for ACEs, $65 \%$ were not confident in screening [10]. Another study surveying general paediatricians $(N=302)$ revealed only $4 \%$ routinely screened for seven
ACEs, 33\% did not usually screen, only $2 \%$ used an ACE screening tool, and $<11 \%$ reported formal training on ACE screening [11].

\section{PERCEPTION AND ATTITUDE OF SCREENING/ PERCEIVED CONTROL OVER SCREENING AND INTERVENTION}

All surveyed physicians believed that ACEs were important in primary health; however, only $5 \%$ reported there was enough time to screen or enough resources to intervene. Inadequate time to complete all health maintenance screening in primary care continues to be an important barrier. Providing ACE screening questionnaires when patients check-in, to be filled out prior to their appointment, may help overcome this barrier. While little research has been done on the feasibility of incorporating ACE screening into Polish paediatric visits, a US study done on screening adults for ACEs with a written questionnaire prior to primary care appointments revealed that all surveyed physicians $(N=111)$ did not believe the screening interfered with the visits [12]. Of the patients screened $(N=27), 91 \%$ that scored $\geq 1$ ACE had an increase in appointment time of $\leq 5$ minutes and only $3 \%$ had an increased time of 10-15 minutes [12]. This supports the time feasibility in ACE screening during primary care appointments. Further research on resources available in the Rzeszów area and compilation into a concise packet for physicians may help overcome intervention barriers. Such interventions include focusing on enhancing modifiable resilience factors such as executive function skills and positive appraisal of self, responsive parenting relationships, maternal mental health, self-care skills and household routines, and helping families and children understand paediatric trauma [13]. While the focus of this study was identifying the prevalence of ACEs and physician screening practices, further research on protective or resilience factors of this population would aid in delineating patients who need intervention and in developing intervention strategies that build on these factors.

\section{PERCEIVED CULTURAL ACCEPTANCE OF SCREENING}

Of the surveyed physicians, $16 \%$ reported that it was not a physician's role to discuss ACEs, with 33\% remaining neutral/unsure. Additionally, 33\% of physicians remained neutral/unsure that ACE screening was appropriate. The above discussions regarding needed development of education for the primary care physician on health promotion and social factors affecting health may also increase acceptance of screening.

\section{PERCEIVED SOCIAL APPROVAL OF SCREENING}

Over half of the surveyed physicians were neutral or unsure if the leadership would approve of routine ACE 
screening. Discussing routine ACE screening with the leadership may establish an environment in which routine ACE screening is more accepted.

\section{STUDY LIMITATIONS}

As the first study done in Poland assessing adverse childhood experiences in Polish paediatric patients and ACE screening practices, this study gives new information and is intended to open discussion and further studies on the topic of ACEs in child health and development. The CYW ACE-Q, while widely used in the US, has not been validated in Poland and may not encompass the ACEs and toxic stress conditions specific to Poland. This study was also limited by a smaller sample size of patients and physicians in Rzeszów and may not be generalisable to the rest of Poland or other areas of Eastern Europe.

The results must be interpreted with caution because the impact of these ACEs cannot be inferred for each patient. Because this study did not screen for protective or resilience factors of the patients, the number of patients screening positive may not reflect the number of patients in need of intervention. Furthermore, the impact of ACEs depends on other factors, including the age of the patient and length of exposure; thus, ACEs between the wide age range of surveyed patients cannot be directly compared. Additionally, the questionnaire assessing provider stress perception was subjective in design, which may affect the accuracy of translation into quantitative data.

\section{CONCLUSIONS}

This study demonstrates a significant prevalence of ACEs within this paediatric sample in Rzeszów and an incremental benefit of supplemental ACE screening to physician perception of family and patient stress. However, larger multi-centre or longitudinal studies are needed to address the limitations of this study. Further research can be used to guide implementation and education of physicians on the practical aspects of ACE screening and intervention strategies.

\section{DISCLOSURE}

The authors declare no conflict of interest.

\section{REFERENCES}

1. Bucci M, Marques SS, Oh D, Harris NB. Toxic Stress in Children and Adolescents. Adv Pediatr 2016; 63: 403-428.

2. Felitti VJ, Anda RF, Nordenberg D, et al. Relationship of Childhood Abuse and Household Dysfunction to Many of the Leading Causes of Death in Adults. Am J Prev Med 1998; 14: 245-258.

3. Bellis MA, Hughes K, Leckenby N, et al. Adverse childhood experiences and associations with health-harming behaviours in young adults: surveys in eight eastern European countries. Bull World Health Organ 2014; 92: 641-655.
4. Makaruk K, Włodarczyk J, Sethi D, et al. Survey of Adverse Childhood Experiences and Associated Health-Harming Behaviours among Polish Students. World Health Organization, 2018.

5. Kaczmarek M, Trambacz-Oleszak S. HRQoL impact of stressful life events in children beginning primary school: results of a prospective study in Poland. Qual Life Res 2017; 26: 95-106.

6. Burke Harris N, Renschler T. Center for Youth Wellness ACE-Questionnaire (CYW ACE-Q Child, Teen, Teen SR). Center for Youth Wellness. San Francisco, CA.

7. Bucci M, Gutierrez L, Koita K, et al. Center for Youth Wellness ACE-Questionnaire User Guide 2015: 2012-2015.

8. Marcinowicz L, Konstantynowicz J, Chlabicz S. The patient's view of the acceptability of the primary care in Poland. Int J Qual Heal Care 2008; 20: 277-283.

9. Pabis M, Wronska I, Slusarska B, Cuber T. Paediatric nurses' identification of violence against children. J Adv Nurs 2011; 67: 384-393.

10. Tink W, Tink JC, Turin TC, Kelly M. Adverse childhood experiences: Survey of resident practice, knowledge, and attitude. Fam Med 2017; 49: 7-13.

11. Kerker BD, Storfer-Isser A, Szilagyi M, et al. Do Pediatricians Ask about Adverse Childhood Experiences in Pediatric Primary Care? Acad Pediatr 2016; 16: 154-160.

12. Glowa PT, Olson AL, Johnson DJ. Screening for Adverse Childhood Experiences in a Family Medicine Setting: A Feasibility Study. J Am Board Fam Med 2016; 29: 303-307.

13. Traub F, Boynton-Jarrett R. Modifiable Resilience Factors to Childhood Adversity for Clinical Pediatric Practice. Pediatrics 2017; 139: e20162569. 